\title{
The Application of the Self-Generation Effect to the Learning of Blissymbols by persons with a Severe Aphasia
}

Priya Rajaram1,*, Erna Alant1,2 \& Shakila Dada 1

1 Center for Augmentative and Alternative Communication, University of Pretoria, South Africa and 2Indiana University, Bloomington, Indiana, USA

\begin{abstract}
This study investigated the application of the self-generation effect to enhance the recognition and retention of Blissymbols in persons with severe aphasia. A 2 X2X3 factorial design of two treatment types (self-generation and non-generation) was used to teach two sets of Blissymbols. These were administered during three training days, between which were withdrawal periods of one day and seven days. Recognition and retention probes were administered at intervals during the training. ANOVA analysis showed that the self-generation treatment produced no immediate recognition advantage; however, better retention of symbol recognition may have occurred over time. Hence, the potential application of the self-generation effect in enhancing the retention of Blissymbols in persons with severe aphasia may warrant further investigation.
\end{abstract}


The self-generation effect (SGE) refers to the finding of superior retention and recall for stimuli constructed or generated by a normal, healthy adult. Memory for stimuli such as words, numbers and pictures were found to be enhanced by the extent to which the individual was involved in its construction (Ghatala, 1981; Jacoby, 1978; McFarland, Frey \& Rhodes, 1980; Peynircioglu, 1989; Slamecka \& Graf, 1978; Tyler, Hertel, McCallum \& Ellis, 1979). Although several studies have shown that individuals with severe aphasia can acquire graphic symbol systems like Blissymbols, further investigation is required to identify the methods that best improve the acquisition, retention and communicative use of such symbol systems (Beck \& Fritz, 1998; Bertoni, Stoffel \& Weniger, 1991; Koul, Corwin \& Hayes, 2005; Koul \& Harding, 1998; Koul \& Lloyd, 1998; McCall, Shelton, Weinrich \& Cox, 2000; Weinrich, Shelton, McCall \& Cox, 1997). This study proposes that the SGE may provide one such option for improving the recognition and retention of Blissymbols.

Research into the SGE has found it to be a robust phenomenon of memory that emerges during free recall and recognition testing (Slamecka \& Graf, 1978). Different types of construction or generation methods have also successfully elicited the SGE through deriving opposites, synonyms, and rhyming words; solving mathematical problems; and completing sentences. The SGE does not appear to be influenced by the type of generation method applied; rather, it emerges during recall or recognition testing when there has been effortful processing and encoding of the to-be-remembered stimuli's attributes at the time of generation (Jacoby, 1978; Ghatala, 1981; Tyler et al., 1979). 
Jacoby (1978) tested the benefits of SGE for word stimuli generated via problem solving. In his first experiment, 18 participants were required to either read or construct member pairs of related words. The subjects were required to generate a word related to a cue word using the letters provided as clues (e.g., dog - b_ $\mathrm{n}_{-}$bone) or to read out the word pairs provided (e.g., sun - shine). Jacoby (1978) reported that subjects recalled more generated words than read words during testing. He attributed this superior recall for the generated word stimuli to SGE.

Using healthy college students, Tyler et al. (1979) compared recall for word stimuli derived from a simple sentence completion task to a more challenging anagram task. In the sentence completion task, the words to be recalled were selected from two words provided to fill in a blank in a sentence (e.g., The girl was awakened by a frightening..... dream or apple). In the anagram task, the participants unscrambled a series of letters to identify a word (e.g., dortoc unscrambled to doctor). The data of Tyler et al. (1979) showed superior recall for word stimuli generated by the more challenging anagram task than by the sentence completion task. Similarly, McFarland, Frey \& Rhodes (1980) also showed that the self-generation factor appeared to increase recall for generated word stimuli. The subjects - normal, healthy college students - either generated a word that rhymed with a given word or determined whether a pair of given words rhymed. Again, a stronger SGE was elicited for the generated items compared with the nongenerated items.

Similarly, other types of stimuli, such as pictures, have also successfully elicited the SGE (Kinjo \& Snodgrass, 2000; Peynircioglu, 1989; Pring, Freestone \& Katan, 1990; Wills, Soraci, Chechile \& Taylor, 2000). Peynircioglu (1989) was the first to report an SGE for pictures in a series of four experiments using normal, healthy college students. In each experiment, the SGE 
was explored under different generation and non-generation conditions, comparing recall for generated and non-generated picture stimuli. The picture generation conditions included drawing pictures by copying a sample picture, drawing a picture using simple written instructions and drawing pictures using connect-the-dot illustrations. Superior memory for the generated pictures was observed in all four experiments.

Pring, Freestone \& Katan (1990) also evaluated the role of SGE with picture stimuli. The participants were blind and sighted children. In this study, the participants recalled different pictures with raised shapes after exposure. In the self-generation condition, participants touched the raised-shape picture then proceeded to generate the picture's name; in the non-generated condition, the labels for the raised-shape pictures were provided. Recall of the generated labels was again superior to recall of the non-generated labels. Similarly, Kinjo \& Snodgrass (2000) also found a generation advantage for picture stimuli when testing for the SGE in normal adults. In this study, the naming of fragmented, incompletely drawn pictures established the generation condition, and the naming of complete pictures established the non-generation condition. Wills et al. (2000) also showed a recall advantage for pictures generated using connect-the-dot illustrations compared with traced or visually scanned pictures.

However, under some circumstances, the SGE may not emerge in typically developing children and adults. Previous studies have shown that it failed to emerge with stimuli that were new, unfamiliar or meaningless (Gardiner \& Hampton, 1985; Lutz, Briggs, \& Cain, 2003; McElroy \& Slamecka, 1982; Nairne, Pusen and Widner, 1985; Nairne \& Widner, 1987; Payne, Neely \& Burns, 1986). Lutz et al. (2003) found a stronger SGE for familiar clichés when contrasted with unfamiliar sentences from textbooks. When studying the SGE in monkeys using photographs as stimuli to contrast, Kornell \& Terrace (2007) were only able to elicit the SGE 
after two weeks of generation training. The subjects were presented with a set of five novel photographs on a touch screen and were expected to touch the photographs in a pre-determined sequence. In the non-generation condition, the subjects received cues or hints about how to perform the touch sequence. In the self-generation condition, the subjects performed the sequence using only trial and error. The subjects' performance levels were better using the non-generation condition during the first three days of training; however, as training continued, the selfgeneration condition started to show higher performance levels. The researchers concluded that "although the active generation of answers during training may result in low initial performance, it enhances long-term retention and transfer" (Kornell \& Terrace, 2007, p. 685).

Despite circumstances when the SGE may not emerge, the application of the SGE to persons presenting with severe aphasia still appears promising due to its successful application in people with other types of acquired brain damage (Barrett, Crucian, Schwartz, \& Heilman, 2000; Chiaravalloti \& DeLuca, 2002; Chiaravalloti, DeLuca, Moore \& Ricker, 2005; Dick \& Kean, 1989; Goverover, Chiaravalloti, Johnston \& DeLuca, 2005; Lengenfelder, Chiaravalloti \& DeLuca, 2003; Lipinska, Backman, Mantyla \& Viitanen, 1994; Michell, Hunt \& Schmitt, 1986; Multhaup \& Balota 1997; O’Brien, Chiaravalloti, Arango-Lasprilla, Lengenfelder \& DeLuca, 2007; Souliez, Pasquier, Lebert, Leconte \& Petit, 1996). Multhaup \& Balota (1997) studied the SGE with three groups, healthy elderly adults, participants with mild Alzheimer-type dementia and participants with very mild Alzheimer-type dementia, using complete and incomplete sentences as stimuli. The generation condition was established by asking the participant to complete a sentence by generating a missing word. In the non-generation condition, the examiner read a sentence to the participant. To test for the SGE, researchers used a forced-choice recognition test and a source monitoring test (Kinjo \& Snodgrass, 2000), which required the 
participant to judge whether the sentences presented were self-generated or examiner-generated. All three participant groups demonstrated superior recall for the generated sentences. Similar results were replicated with people with mild to moderate dementia by Souliez et al. (1996), Lipenska et al. (1994) and Barrett et al. (2000).

Chiaravalloti \& DeLucca (2002) studied self-generation as a means of maximizing learning in multiple sclerosis (MS), a condition where memory appears to be the most consistently identified cognitive dysfunction. Because people with MS show a deficit in acquiring new information, it was expected that the SGE could provide a way for them to generate their own to-be-remembered stimuli. The participants were required to recall and recognize a list of words presented in complete and incomplete written sentences. In the nongeneration condition, the to-be-recalled word was underlined in the written sentence. In the selfgeneration condition, incomplete sentences were used; the to-be-recalled word was omitted, and participants were required to generate the missing word to complete the sentence. The results showed superior recall and recognition for the generated words in both the control group and the MS group.

O’Brien et al. (2007) investigated the SGE persons presenting with traumatic brain injury (TBI) and multiple sclerosis. The researchers also aimed to describe the benefits of the SGE in participants with different types of cognitive impairment. Participants showed a strong SGE for words in both the TBI and MS groups, confirming that individuals with deficits in multiple cognitive domains after brain injury may also benefit from the SGE.

Dick \& Kean (1989) also investigated the SGE in patients with mild to moderate Alzheimer-type dementia and a control group of adults without dementia, using sentences as stimuli. In the generated condition, participants generated the last word in the sentence. Some 
letters of the word were provided to constrain responses. In the non-generated condition, the last word was presented in a larger font, requiring the participant only to read. The stimuli presentation was repeated three times. The test procedures included a free recall test and a source monitoring test including word stem completion. The results showed an SGE for the healthy control group, but no SGE for the participants with dementia. Mitchell, Hunt \& Schmitt (1986) found similar results, arguing that because semantic memory is disrupted in Alzheimer's typedementia, they did not expect to find an SGE in these participants. Such results provided support for a semantic activation theory for the SGE.

Hence, this study investigated whether the SGE could be used to enhance the recognition and retention of Blissymbols in persons presenting with severe aphasia. The method for eliciting the SGE in the present study was guided by the prominent semantic activation theory, which states that the effortful act of generating a stimulus item, such as a word or a picture, activates its location in the lexical/semantic network and enhances its retrieval from memory (Graf, 1980; McElroy \& Slamecka, 1982; Nairne et al., 1985; Payne et al., 1986). Early studies by Jacoby (1978) and Slamecka \& Graf (1978) showed the SGE to be a function of the individual's processing of stimuli in an elaborate way in the generation condition and in an unelaborated, non-distinctive manner in the non-generation condition. In the present study, the generation condition involved completing connect-the-dot illustrations. The to-be-recognized Blissymbols were converted into connect-the dot illustrations, as used by Peynircioglu (1989) and Wills et al. (2000). Participants generated the to-be-remembered Blissymbols by connecting the dots. In contrast, the non-generation condition involved a paired-association activity in which the participant was visually exposed to the completed Blissymbol, and its referent was named verbally. Hence, the participant's generation of the connect-the-dot Blissymbol illustrations 
involved more cognitive effort than the non-generation condition. Completing the dot illustrations led to the active encoding and effortful processing of the Blissymbols at the time of generation, eliciting the SGE memory advantages during recognition and retention testing.

As Kornell \& Terrace (2007) and Lutz et al. (2003) suggested, the SGE may emerge over time when new, meaningless or unfamiliar stimuli (such as the Blissymbols used in this study) are used. In the current study, training and testing for the emergence of the SGE occurred across three different experimental sessions spread over a period of time, with two withdrawal periods. The first withdrawal period of one day followed experimental session one and the second withdrawal period of seven days followed experimental session two. Recognition and retention probes were used as two types of testing probes. The recognition probes occurred directly after training, thereby identifying the possible immediate recognition benefits of the SGE. The retention probes were administered after the withdrawal periods. These probe measures permitted probing for the emergence of the SGE directly after training and also assessed the influence of the SGE on the retention of previously recognized symbols over time.

\section{Method}

\section{Study Design}

A 2 (treatment approaches) x 2 (symbols sets) x 3 (training days) factorial arrangement with an alternating treatments design was used. All factors were within subjects, in which each participant received all treatments, but in an alternating manner. The order of treatment presentation was counterbalanced within participants across their treatment sessions. For each participant, both treatments were applied consecutively during one treatment session, with the order of presentation alternating in subsequent sessions. The treatments were applied to two 
different but equivalent to-be-learned symbol sets. The daily delivery of each treatment was followed by a measurement of the recognition of the Blissymbols. Table 1 shows the alternations of treatments (T1 self-generation and T2 non-generation) and symbol sets ( 1 1 and S2) within and between participants.

[Insert Table 1 About Here]

\section{Participants}

Eight participants with severe aphasia following a cerebral vascular accident (CVA) were included in the study. A group of 23 prospective candidates was recruited from two rehabilitation hospitals via referrals from the resident speech-language therapist. Potential participants were screened to confirm whether they met the predetermined participant selection criteria, as listed in Table 2. The presence of an aphasia was confirmed by a neurological examination conducted by the attending neurologist. The neurologist also confirmed the absence of co-existing cognitive disorders, as documented in the clinical case notes from the referral source. To determine whether the prospective candidates met the participant selection criteria, a set of screening tests was administered, including (a) the Boston Diagnostic Aphasia Examination (BDAE) (Goodglass \& Kaplan, 2001) to confirm the aphasia severity level; (b) a pointing skills test to ensure that the participant could point to a named picture referent (c) an informal receptive language test, in which the prospective participant was asked to select (by pointing) a verbally named referent from one of seven $2 \times 3$ grid boards of simple picture representations of the 28 symbol referents in this study and six foils; ; (d) a connect-the-dot execution test to ensure that the participant could complete the self-generation task; (e) a visual-discrimination test, in which the participant had to match a set of Blissymbol cards to its matching partner on a $2 \mathrm{X} 3$ grid board, ensuring that the 
participant could visually discriminate between symbols; and (f) an informal assessment of attention level, which was performed during the BDAE administration to confirm that the prospective participant would not fatigue during the experimental tasks. Informed written consent was obtained from the participant or the participant's caregiver. Of the eight participants, four presented with severe global aphasia, and four presented with severe Broca's aphasia, as determined by their performance on the BDAE (Goodglass \& Kaplan, 2001). Table 3 provides a profile of the final eight participants who passed the screening procedure and were included in the study.

\section{[ Insert Table 2 and Table 3 About Here]}

\section{Materials}

Symbol sets

Two equivalent Blissymbol sets were developed to be counter-balanced within participants and between the two treatment approaches to avoid an exposure bias. The main equivalency variables used to balance the symbol sets were translucency, complexity, familiarity and frequency of use. These variables have been shown to influence the acquisition of Blissymbols in adults and children (Fuller, 1997; Fuller \& Lloyd, 1987; Koul \& Lloyd, 1998). Fuller's (1997) set of 40 Blissymbols allocated into four groups, including high translucency-high complexity, high translucency-low complexity, low translucency-high complexity, and low translucency-low complexity, were used. To verify the ratings for translucency, familiarity and frequency of use, Fuller's (1997) 40 symbols (Fuller, 1997) were re-rated by 18 South African undergraduate students using Likert scales. Fuller \& Lloyd's (1987) complexity values were used to determine the symbol's complexity rating. The Friedman two-way Analysis of Variance test 
was used to analyze the re-rated symbols. This resulted in eight symbols being rejected from the Fuller's (1997) list of 40 symbols because the translucency re-ratings differed significantly from Fuller's (1997) ratings.

The remaining 32 symbols were randomly allocated into two symbol sets, ensuring that each set had an equivalent spread of symbols from each group. These two sets of symbols (set 1 , set 2) were then rated for their equivalency by eleven raters to ascertain whether the symbols were balanced within each set for translucency, frequency of use and familiarity. To rate translucency, the raters were presented with a written record sheet that presented pairs of symbols, one symbol from set 1 and one symbol from set 2 , each on a new line on the sheet. These symbol pairs were randomly allocated from set 1 and set 2 and had the same translucency values. The raters evaluated the equivalency of the pair of symbols in terms of translucency. To rate the symbol pairs' frequency of use and familiarity, the raters were presented with randomly allocated pairs of referents and were required to rate their equivalency using a Likert scale. This procedure resulted in the exclusion of an additional four symbols. A total of 28 symbols were available for allocation into the final two symbol sets. The 28 symbols were randomly divided into two sets of 14 symbols, ensuring an equal spread of symbols from each of Fuller's (1997) translucencycomplexity groupings.

Connect-the-dot illustrations

To successfully elicit the SGE, the participants were required to construct or generate the to-be-recognized Blissymbols. Hence, the Blissymbols were converted into connect-the-dot illustrations. The participants were simultaneously presented with a complete symbol card and a connect-the-dot illustration of the Blissymbol. The participants were then required to generate the 
given complete Blissymbol by completing the connect-the-dot illustration in a way that produced a symbol matching the sample. The provision of the sample Blissymbol was important. Given the severe linguistic deficits associated with a severe aphasia, the participants would not have been able to connect consecutive numbered connect-the-dot illustrations like those used by Wills et al. (2000) when they investigated the role of "aha" effect in picture generation. To standardize each dot illustration of the Blissymbols, the number of dots used per symbol was correlated to the symbol's complexity value (Fuller and Lloyd, 1987). The dot illustrations (shown in Appendix B) were drawn by a professional illustrator. A large diameter $(1 \mathrm{~mm})$ was selected for each dot. Each stimulus card was approximately $8.5 \mathrm{~cm}$ by $12 \mathrm{~cm}$ and included the dot illustration together with its written referent.

\section{Procedure}

Table 4 shows the timing and the procedural format for each experimental session. Each of the eight participants took part in three individual experimental sessions (E1 - Day 1, E2 - Day 3, and E3 - Day 11), with one day between E1 and E2 and seven days between E2 and E3. As shown in Table 1, the selection of symbol sets and treatments was carefully counter-balanced and alternated within participants to avoid order or presentation effects. Table 4 also shows the teaching criteria, as determined by a pilot study, which prescribed the number of exposure blocks for the symbol sets used during each experimental session.

\section{[Insert Table 4 About Here]}

During each of the three experimental sessions (E1, E2 and E3), the two treatment approaches (the self-generation treatment and the non-generation treatment) were administered. In E1, training using one of the treatment approaches was administered, followed directly by a 
recognition probe measure. The training in E1 included two blocks of exposure to the symbol set. The recognition probe measured how many symbols the participant learned to recognize after that particular treatment approach. After a 5-minute rest period, the next treatment approach was started, followed immediately by a recognition probe. Again, two blocks of exposures to the symbol set were used during the training.

The format for the subsequent experimental sessions (E2 and E3) on Day 3 and Day 11 changed. Prior to the start of the session, the retention probes were administered, to measure symbol retention levels following the one-day and seven-day withdrawal periods. The number of training exposure blocks was decreased to one.

\section{Description of the Training}

During training using the self-generation treatment (T1), the participant was instructed to complete the dot illustration, establishing the effortful processing required for the SGE to emerge. A complete sample symbol card (including the referent's written gloss) was in view to guide the participant in completing the dot pictures. Although the researcher did not point out construction errors, the participants were informed that they could correct their own errors. The symbol referent was named out loud three times: when the sample symbol was presented, during the generation task, and upon completion of the generation task.

In the non-generation condition (T2), the participant was presented with the complete symbol card (including the referent's written gloss) and was instructed to study the symbol card, which represented the named referent. The symbol referent was named out loud three times at the same intervals used during T1. Under both conditions, the participant was reminded of the recognition test to follow, and the exposure time to each symbol was kept consistent (a maximum 
of one minute to study or construct the symbol, as determined during the pilot study). As specified by the teaching criterion determined during the pilot study, two blocks of training (exposures to the symbol set) were conducted on E1 (Day 1), and one block of training was conducted on E2 (Day 3) and E3 (Day 11).

\section{Scoring}

Two types of probe measures were used: recognition probes (RP) and retention probes (RTP). The timing of the probe measures is shown in Table 4. The recognition probes occurred directly after the training on a treatment approach, was completed. These recognition probes measured the number of symbols correctly recognized directly after training to determine the success of the approach. Because each symbol set contained 14 symbols, the recognition probes measured recognition of the 14 symbols in the set.

The retention probes (RTP) measured retention of the Blissymbols after the withdrawal periods across both treatment approaches and for both symbol sets; hence, the retention probes tested for all 28 symbols from symbol sets 1 and 2 . This helped to identify whether any one treatment approach produced better retention of symbols over time. The retention probes were conducted at the start of training days E2 (Day 3) and E3 (Day 11).

During the recognition probes, two $4 \mathrm{X} 2$ symbol boards were presented individually: the first with eight symbols and the second with six symbols. The arrangement of the symbols on the boards was randomly changed for each recognition probe. The researcher instructed the participant to point to randomly named symbols. During the retention probes, four $4 \mathrm{X} 2$ symbol boards were presented individually; the first three boards had eight symbols, and the fourth had four symbols. The grids included all 28 symbols from sets 1 and 2, which were randomly placed. 
Again, the symbol arrangement on the board was randomly changed for each retention probe. The participant was instructed to point to the symbol that matched the named referent.

During both types of probes, a response was scored only if the participant pointed to the symbol that matched the name called by the experimenter. If the response was incorrect, it was not brought to the participant's attention, and the experimenter proceeded to name the next referent. No verbal praise was used. The total number of correct responses was tallied. A maximum score of 14 for the recognition probes and 28 for the retention probes was possible, and each participant had six recognition probe and two retention probe scores.

\section{Reliability}

An inter-rater test was conducted to confirm the reliability of the training procedures and the scoring. Two qualified, practicing speech-language therapists were used as raters. This test required the rater to view three randomly selected, video-recorded experimental sessions. A checklist was developed to allow the raters to assess the integrity of the experimental procedures. The checklist focused on rating the equivalency of the training procedures across treatment types and the accuracy of the probe scores. The scores were tallied, and favorable inter-rater agreement levels of $80 \%$ to $100 \%$ were obtained (Maxwell \& Satake, 2006). This exceeds the $70 \%$ interrater agreement level recommended by Macmillian \& Schumacher (2001) for good inter-rater reliability.

\section{Data Analysis}

A $2 \mathrm{X} 2 \mathrm{X} 3$ (treatment approaches $\mathrm{X}$ symbol sets $\mathrm{X}$ training days) analysis of variance (ANOVA) was performed on the participants' recognition and retention scores. A repeated ANOVA was used to determine whether the treatment approach, symbol set or an interaction 
between the two influenced the retention of recognition over time. Post hoc tests using least square means were performed to explore any significant differences in the retention scores. Cohen's effect size $d$ (Cohen, 1988) was used to determine the strength of the significant effects identified. A medium effect size was assumed to be between 0.06 and 0.14 , and a large effect size was assumed to be $>0.157$. All statistical testing took place at a 0.05 significance level.

\section{Results}

The ANOVA showed no significant interaction between treatments, symbols sets and training days when the recognition scores were analyzed. Hence, there seemed to be no immediate benefit from the self-generation treatment $(\mathrm{F}(2,36)=1.38, \mathrm{p}=.26, d=.07)$. Both treatments resulted in similar recognition levels $(\mathrm{T} 1$ - self generation $\mathrm{M}=9.62, \mathrm{SD}=2.14 ; \mathrm{T} 2$ - nongeneration $\mathrm{M}=8.53, \mathrm{SD}=3.01$ ). The repeated ANOVA showed a significant interaction between treatment and set regarding the difference in retention between training days E2 (Day 3) and E3 (Day 11) $(\mathrm{F}(1,12)=14.99, \mathrm{p}=.00, d=.19)$. This difference in retention scores between E2 (Day 3) and E3 (Day 11) was further investigated using least square means t-tests. The results are reported in Table 5, which shows the mean retention scores for each treatment and symbol set for E2 (Day 3) and E3 (Day 11), respectively. It also shows the mean difference in retention levels between E2 (Day 3) and E3 (Day 11) for each treatment. The largest significant mean difference in retention levels between E2 (Day 3) and E3 (Day 11) was seen for Treatment 2 (nongeneration) with set $1(\mathrm{M}=-4.02, \mathrm{SD}=1.41)$. As shown in Figure 1 , there was a reduction in the number of symbols recognized on training day E2 (Day 3) compared to training day E3 (Day 11) when using Treatment 2 (non-generation) and set 1. For T1 (self-generation), similar numbers of symbols were recognized on training day E2 (Day 3) $(\mathrm{M}=9.33, \mathrm{SD}=1.96)$ as in the retention probe on E3 (Day 11) $(\mathrm{M}=9.54, \mathrm{SD}=1.87)$. However, for T2 (non-generation treatment), more 
symbols were recognized on E2 (Day 3) $(\mathrm{M}=11.56, \mathrm{SD}=2.12)$ than during the retention probe on Day $11(\mathrm{M}=7.57, \mathrm{SD}=3.5)$. However, this trend was restricted to symbol set 1 and did not occur at a significant level for symbol set 2.

\section{[Insert Table 5 and Figure 1 About Here]}

\section{Discussion}

The study confirms that participants with severe global aphasia and severe Broca's aphasia do have the ability to recognize Blissymbols. This is consistent with previous studies showing that augmentative and alternative communication (AAC) may be a viable method for establishing communication in these individuals (Koul, Corwin \& Hayes, 2004; Koul \& Harding, 1998; Koul \& Lloyd, 1998; McCall, Shelton, Weinrich \& Cox, 2000). However, there seems to be no obvious, immediate benefit in the generation of to-be-recognized symbols. The theory of semantic activation may be the most likely explanation for why the SGE failed to emerge during the recognition probes. The semantic activation theory for the SGE suggests that the act of generating allows greater processing of the stimulus item's attributes, thereby facilitating activation of the item's location in semantic memory to a greater degree than a non-generation task (Graf, 1980; McElroy \& Slamecka, 1982; Nairne et al., 1985; Payne et al., 1986). It may be that the connect-the-dot activity used for the generation condition in the present study may have been distracting and did not allow for the optimal processing of the semantic association between the symbol and its referent, which would explain why semantic activation did not occur and why the SGE failed to emerge during recognition testing.

The stimuli used may provide another possible explanation for why the SGE did not emerge. Some SGE studies have not shown an SGE for non-words, unfamiliar and non- 
meaningful stimuli (Gardiner \& Hampton, 1985; Nairne, Pusen and Widner, 1985; Nairne \& Widner, 1987). The to-be-recognized Blissymbols were new and non-meaningful picture stimuli when first presented to the participants, and they may have failed to attach meaning to the Blissymbols on initial exposure. The contention that the SGE may not emerge with new, unfamiliar stimuli has also been cited by other studies that failed to elicit the SGE with neurologically impaired populations (Dick \& Kean, 1989; Mitchell, Hunt \& Scmitt, 1986; Souliez, Pasquier, Lebert, Leconte \& Petit; 1996).

Interestingly, the SGE did seem to emerge over time, albeit only on one symbol set. Following the seven-day withdrawal period, the self-generation treatment seemed to support more retention of the symbols. This points to the possible robustness of the memory enhancing effect caused by the self-generation treatment. Interestingly, the similar increased, long-term retention benefit for generated stimuli was also reported by Kornell \& Terrace (2007) when they studied the SGE in monkeys.

The emergence of the SGE over time may also provide further support for the semantic activation theory for the SGE. It is possible that following repeated exposures to the Blissymbols during the training sessions, the participants may have started to develop a conceptual representation for the Blissymbols and begun to attach meaning to the symbols. Hence, an SGE was starting to emerge during the final training session. Because the SGE has been shown to be ineffective for unfamiliar stimuli, heightened exposure during training may have also increased the participants' familiarity with the Blissymbols, causing the SGE to emerge.

Evidence supporting the emergence of the SGE over time was only observed in one part of the data set. The current study's methodology may have contributed to this skewing of the data. 
First, the influence of cumulative learning must be considered. Although a rigorous equivalency procedure was used to equate the two symbol sets used, repeated exposures to the same two sets of stimuli over the three training days may have resulted in cumulative learning, and some symbols may have been learned better than others. However, if cumulative learning did occur, a steady increase in recognition levels across both treatment approaches early in the training would have been seen. Because participants appeared to benefit from the exposures and the construction of the symbols (i.e., the self-generation condition) and not from exposures alone (i.e., the nongeneration condition), it is more likely that the self-generation effect resulted in superior retention over time.

The inclusion of both Broca's aphasics and global aphasics in the study may have also contributed to a restriction in the findings. Although there was an equal split between these two types of severe aphasias and participants were matched across four variables (time of onset, severity, lesion site and education), there was significant heterogeneity between groups. These aphasic syndromes may respond differently to the treatments, thus restricting the findings to one symbol set.

\section{Conclusion}

The recognition scores indicate that there are no overall differences in recognition levels for Blissymbols using either treatment approaches. This is consistent with previous research findings showing no SGE for new, non-meaningful and unfamiliar material. The finding of similar recognition levels across treatments during the recognition testing also provided support for a semantic activation theory for the SGE. Because the Blissymbols were new, non-meaningful stimuli, no semantic associations were made initially. However, the results did show that there 
was a statistically significant difference in recognition levels between the self-generation and nongeneration approach following the seven-day withdrawal period. The self-generation treatment appeared to produce better retention of recognition levels over time. The stimuli became more familiar to the participants upon repeated exposure; thus, semantic associations between the symbol and its referent may have become more apparent, which allowed the SGE to emerge over time.

\section{Critical Evaluation and Recommendations for Future Research}

The inclusion of the retention probe measures was a strength of this study as they allowed for the emergence of the SGE over time. If the retention probes had not been included, the emergence of the SGE over time may have been missed. Although a rigorous equivalency procedure was used to develop the two symbol sets alternated within participants, repeated exposures to the same two sets of stimuli over the course of the training may have also resulted in cumulative learning of the to-be-recognized Blissymbols. Hence, some of the small benefit that was observed for the SGE over time remains questionable. The use of more difficult test probes, such as free recall of the generated symbols or comprehension testing for the generated symbols, may help to reduce the effects of repeated exposures to the same stimuli sets. The use of more challenging testing formats may increase the memory demands placed on the participant, resulting in the earlier emergence of the memory-enhancing effects associated with the SGE. Because the unfamiliarity of the Blissymbols could have influenced the emergence of the SGE, future research should evaluate how people with severe aphasia who have already been exposed to Blissymbols may perform when using self-generation. 


\section{References}

Barrett, A. M., Crucian, G. P., Schwartz, R. L., \& Heilman, K. M. (2000).Testing memory for self-generated items in dementia: Method makes a difference. Neurology, 54(6), 1258-1264.

Beck, A.R., \& Fritz, H. (1998). Can people who have aphasia learn iconic codes? Journal of Augmentative \& Alternative Communication, 14, 184 -196.

Bertoni, B., Stoffel, A., \& Weniger, D. (1991). Communicating with pictographs: A graphic approach to the improvement of communicative interactions. Aphasiology, 5, 341-353.

Chiaravalloti, N. D., \& Deluca, J. (2002). Self-generation as a means of maximizing learning in multiple sclerosis: An application of the generation effect. Archives of Physical Medicine \& Rehabilitation, 83, 8, 1070-1079.

Chiaravalloti, N. D., DeLuca, J., Moore, N. B., \& Ricker, J. H. (2005). Treating learning impairments improves memory performance in multiple sclerosis: A randomized clinical trial. Multiple Sclerosis, 11, 1, 58-68.

Craik, F. I. M., \& Lockhart, R.S. (1972). Levels of processing: A framework for memory research. Journal of Verbal Learning and Verbal Behaviour, 11. 671-684.

Dick, M.B., \& Kean, M.L. (1989). Memory for internally generated words in Alzheimer-type dementia: Breakdown in encoding \& semantic memory. Brain \& Cognition, 9, 1, 88-108.

Funnel, E., \& Allport, A. (1989). Symbolically speaking: communicating with Blissymbols in aphasia. Aphasiology, 3, 279-300. 
Fuller, D.R. (1997). Initial study into the effects of translucency and complexity on the learning of Blissymbols by children and adults with normal cognitive abilities. Augmentative \& Alternative Communication, 13, 1, 30-39.

Fuller, D. R., \& Lloyd, L. L. (1987). A study of physical and semantic characteristics of a graphic symbol system as predictors of perceived complexity. Augmentative and Alternative Communication, 3, 26-35.

Gardiner, J.M., \& Hampton, J.A. (1985). Semantic memory \& the self-generation effect: some tests of the lexical activation hypothesis. Journal of Experimental Psychology: Learning, Memory \& Cognition, 11, 4, 732-741.

Gardiner, J.M., \& Rowley J.M.C. (1984). A generation effect with numbers rather than words. Memory \& Cognition, 12, 443-445.

Ghatala, E.S. (1981). The effect of internal generation of information on memory performance. American Journal of Psychology, 94, 3, 443-450.

Glisky, E.L. \& Rabinowitz, J.C. (1985). Enhancing the self generation effect through repetition of operations. Journal of Experimental Psychology: Learning, Memory \& Cognition, 11, 2, 193-205.

Goodglass, H., Kaplan, E., \& Barresi, B. (2001). The Boston Diagnostic Aphasia Examination. $3^{\text {rd }}$ edition. Lippincott Williams \& Wilkins.

Goverover, Y., Chiaravalloti, N., Johnston, M., \& DeLuca, J. (2005). Self-generation to improve learning of everyday functional tasks in multiple sclerosis \& traumatic brain injury. Journal of the International Neuropsychological Society, 11 (Suppl. 1), 49-59. 
Graf, P.(1980).Two consequences of generating: Increased inter \& intraword organization of sentences. Journal of Verbal Learning \& Verbal Behaviour, 19, 316-327.

Hegde, M.N. (2003). Clinical research in communication disorders. Principles \& strategies. $3^{\text {rd }}$ edition. Texas: Pro-Ed.

Hirshman, E,. \& Bjork, R.A. (1988). The generation effect: Support for a two-factor theory. Journal of Experimental Psychology: Learning, Memory, and Cognition, 14. 484-494.

Jacoby, L.L. (1978). On interpreting the effects of repetition: Solving a problem versus remembering a solution. Journal of Verbal Learning \& Verbal Behaviour, 17, 649-667.

Johns, E.E., \& Swanson, L.G. (1988). The generation effect with nonwords. Journal of Experimental Psychology: Learning, Memory and Cognition, 14, 180-190.

Kinjo, H., \& Snodgrass, J.G. (2000). Does the generation effect occur for pictures. American Journal of Psychology, 113 (1), 95-121.

Kornell, N., \& Terrace, H.S. (2007). The generation effect in monkeys. Psychological Science, 18 (8), 682-685.

Koul, R.K., Corwin, M., \& Hayes, S. (2005). Production of graphic symbol sentences by individuals with aphasia: Efficacy of a computer-based augmentative \& alternative communication intervention. Brain \& Language, 92 (1), 58-77.

Koul, R.K., \& Harding, R. (1998). Identification and production of graphic symbols by individuals with aphasia: Efficacy of a software application. Journal of Augmentative \& Alternative Communication, 14, 11-23. 
Koul, R.K., \& Lloyd, L.L. (1998). Comparison of graphic symbol learning in individuals with aphasia and right hemisphere brain damage. Brain \& Language, 62, 394-421.

Lane, V.W., \& Samples, J.M. (1981). Facilitating communication skills in adult aphasics: Application of Blissymbolics in a group setting. Journal of Communication Disorders, 14, 157167.

Lengenfelder, J., Chiaravalloti, N. D., \& DeLuca J. (2003). Examining the generation effect in individuals with traumatic brain injury. Journal of the International Neuropsychological Society, 8, 173-184.

Lipinska, B., Backman, L., Mantyla, T., \& Viitanen, M. (1994). Effectiveness of self-generated cues in early Alzheimer's disease. Journal of Experimental \& Clinical Neuropsychology, 16 (6), 809-819.

Lutz, J., Briggs, A \& Cain, K. (2007). An examination of the value of the generation effect for learning new material. Journal of General Psychology, 130 (2), 171-187.

McMillan, J. H., \& Schumacher, S. (2001). Research in education: A conceptual introduction (5th Ed.). New York, NY: Longman.

Maxwell, D.L., \& Satake, E. (2006). Research and statistical methods in communication sciences and disorders. New York: Thomson Delmar Learning.

McCall, D., Shelton, J.R., Weinrich, M., \& Cox, D. (2000). The utility of computerized visual communication for improving natural language in chronic global aphasia: Implications for approaches to treatment in global aphasia. Aphasiology, 21 (8), 795-826. 
McDaniel, M.A., Riegler, G.L. and Waddill, P.J. (1990). Generation effects in free recall: Further support for a three-factor theory. Journal of Experimental Psychology: Learning, Memory, and Cognition, 16, 789-798.

McElroy, L.A. \& Slamecka, N.J. (1982). Memorial consequences for generating nonwords: Implications for semantic-memory interpretations of the self generation effect. Journal of Verbal Learning \& Verbal Behavior, 21, 249-259.

McFarland C.E., Frey, T.J. \& Rhodes, D.D. (1980). Retrieval of internally versus externally generated words in episodic memory. Journal of Verbal Learning \& Verbal Behaviour, 19, 210225.

Mitchell, D.B., Hunt, R.R., \& Schmitt, F.A. (1986). The generation effect and reality monitoring: Evidence from dementia and normal aging. Journal of Gerentology. 41 (1), 79-84.

Multhaup, K. S., \& Balota, D. A. (1997). Generation effects \& source memory in healthy older adults and in adults with dementia of the Alzheimer type. Neuropsychology, 11 (3), 382-391.

Nairne, J.S., Pusen, C., \& Widner, R.L. (1985). Representation in the mental lexicon: Implications for theories of the self generation effect. Memory \& Cognition, 13, 2, 183-191.

Nairne, J.S., \& Widner, R.L. (1987). Generation effects with nonwords: the role of test appropriateness. Journal of Experimental Psychology: Learning, Memory, \& Cognition, 3, 1, 164171.

O’Brien, A., Chiaravalloti, N., Arango-Lasprilla, J.C., Lengenfelder, J., \& DeLuca, J. (2007). An investigation of the differential effect of self-generation to improve learning and memory in multiple sclerosis and traumatic brain injury. Neuropsychological Rehabilitation, 17 (3), 273-292. 
Payne, D.N., Neely, J.H. \& Burns, D.J. (1986). The self-generation effect: further tests of the lexical activation hypothesis. Memory \& Cognition, 14 (3), 246-252.

Peynircioglu, Z.F. (1989). The generation effect with pictures and nonsense figures. Acta Psychologica, 70, 153-160.

Pring, L., Freestone, S.F., \& Katan, S.A. (1990). Recalling pictures and words: Reversing the generation effect. Current Psychology: Research \& Reviews, 9 (11), 35-45.

Slamecka, N.J., \& Graf, P. (1978). The self-generation effect: Delineation of a phenomenon. Journal of Experimental Psychology: Human Learning \& Memory, 4, 592-604.

Souliez, L., Pasquier, F., Lebert, F., Leconte, P., \& Petit, H. (1996). Generation effect in short term verbal and visuospatial memory: Comparisons between dementia of the Alzheimer type and dementia of the frontal lobe type. Cortex, 32, 347-356.

Tyler, S.W., Hertel, P.T., McCallum M.C., \& Ellis, H.C. (1979). Cognitive effort and Memory. Journal of Experimental Psychology: Human Learning \& Memory, 4, 592-604.

Wills, W.T., Soraci, S.A, Chechile, R.A., \& Taylor, H.A. (2000). “Aha” effects in the generation of pictures. Memory \& Cognition, 28 (6), 939-948.

Weinrich, M., Shelton, J.R., McCall, D., \& Cox, D.M. (1997). Generalization from single sentence to multisentence production in severely aphasic patients. Brain \& Language, 58, 327352. 
Table 1

Format of training during experimental sessions: list of alternations between symbol sets (S1, S2) and treatments (T1 self-generation and T2 non-self-generation)

Participant Experimental

Experimental

Experimental

session E1

session E2

session E3

Day 1

Day 3

Day 11

\begin{tabular}{llll}
\hline 1,5 & S1T1 & S1T2 & S1T1 \\
& S2T2 & S2T1 & S2T2 \\
& S1T2 & S1T1 & S2T1 \\
3,7 & S2T1 & S2T2 & S1T2 \\
& S2T1 & S2T2 & S2T1 \\
& S1T2 & S1T1 & \\
& S1T2 & & S2T1 \\
& S2T1 & S1T1 & S1T2 \\
\hline
\end{tabular}


Table 2

Participant selection criteria

Criteria

The etiology of the severe aphasia was confined to a cerebral vascular accident (CVA).

A unilateral left-sided lesion either caused by infarction or an ischemic episode, confirmed by a brain CT scan.

A minimum of one year post-CVA onset.

Adequate receptive language skills.

No uncorrected peripheral vision or visual field deficits or hearing deficits.

Ability to sustain attention for 30 minutes.

Adequate pointing skills, as determined by the pre-experimental screening tests.

No visual discrimination deficits, as confirmed during pre-experimental screening tests.

Ability to complete a connect-the-dot picture of a predetermined number of dots in an allotted time frame using either the right hand or the left hand in the presence of a right-sided hemiplegia or hemiparesis.

No previous exposure to AAC training. 
Table 3

Description of participants

\begin{tabular}{lcccccccc}
\hline Participant & 1 & 2 & 3 & 4 & 5 & 6 & 7 & 8 \\
& & & & & & & & \\
\hline Age & 44 & 42 & 78 & 68 & 57 & 61 & 56 & 48 \\
Months postonset & 40 & 16 & 19 & 25 & 14 & 16 & 18 & 17 \\
Type of aphasia & $\mathrm{G}$ & $\mathrm{G}$ & $\mathrm{G}$ & $\mathrm{G}$ & $\mathrm{B}$ & $\mathrm{B}$ & $\mathrm{B}$ & $\mathrm{B}$ \\
Gender & $\mathrm{M}$ & $\mathrm{M}$ & $\mathrm{M}$ & $\mathrm{M}$ & $\mathrm{M}$ & $\mathrm{M}$ & $\mathrm{F}$ & $\mathrm{M}$ \\
BDAE expressive score & 10 & 0 & 10 & 13 & 23 & 10 & 47 & 37 \\
BDAE receptive score & 13 & 30 & 10 & 10 & 30 & 40 & 57 & 47 \\
BDAE severity rating & 1 & 1 & 1 & 1 & 2 & 2 & 2 & 2
\end{tabular}

Note. $\mathrm{G}=$ Global aphasia, $\mathrm{B}=$ Broca's aphasia

BDAE severity rating reported in percentiles. Definitions: 1) All communication is through fragmentary expression, with a great need for inference, questioning and guessing by the listener. The range of communication is limited, and the listener carries the burden of conversation; 2) Conversation about familiar subjects is possible with help from the listener. Frequent failures to convey ideas, but the patient shares the burden of communication. 
Table 4

Format for experimental (E) sessions conducted during three training days

Participant

$\begin{array}{ccc}\text { Experimental } & \text { Experimental } & \text { Experimental } \\ \text { session E1 } & \text { session E2 } & \text { session E3 } \\ \text { Day 1 } & \text { Day 3 } & \text { Day 11 } \\ & \text { 1-day withdrawal } & \text { 7-day withdrawal }\end{array}$

$1,2,3,4,5,6,7,8 \quad$ Training (two blocks) $\quad$ Retention probe $\quad$ Retention probe

Recognition probe Training (one block) Training (one block)

REST Recognition probe Recognition probe

Training (two blocks)

REST

REST

Recognition probe Training (one block) Training (one block)

Recognition probe Recognition probe 
Table 5

Results of the post hoc tests showing the mean $(M)$ retention scores and the mean difference in retention scores between training days E2 (Day 3) and E3 (Day 11) for Treatment 1(selfgeneration) and Treatment 2 (non-generation)

\begin{tabular}{|c|c|c|c|c|c|c|c|}
\hline \multirow[t]{2}{*}{ Treatment } & \multirow[t]{2}{*}{$\begin{array}{c}\text { Symbol } \\
\text { set }\end{array}$} & \multicolumn{2}{|c|}{ Mean retention score } & \multicolumn{2}{|c|}{$\begin{array}{c}\text { Mean retention score } \\
\text { E3 (Day 11) }\end{array}$} & \multicolumn{2}{|c|}{$\begin{array}{l}\text { Mean difference } \\
\text { between E2 (Day }\end{array}$} \\
\hline & & $\mathrm{M}$ & $\mathrm{SD}$ & $\mathrm{M}$ & $\mathrm{SD}$ & $\mathrm{M}$ & $\mathrm{SD}$ \\
\hline$\overline{\mathrm{T} 1}$ & $\mathrm{~S} 1$ & 9.3 & 1.96 & 9.5 & 1.87 & 0.16 & 0.98 \\
\hline $\mathrm{T} 1$ & $\mathrm{~S} 2$ & 10.5 & 0.71 & 9.0 & 0.00 & -1.5 & 0.71 \\
\hline $\mathrm{T} 2$ & $\mathrm{~S} 1$ & 11.5 & 2.12 & 7.5 & 3.53 & $-4.0 *$ & 1.41 \\
\hline $\mathrm{T} 2$ & $\mathrm{~S} 2$ & 8.2 & 3.76 & 8.6 & 2.94 & 0.5 & 1.76 \\
\hline
\end{tabular}

Note. *Significant mean difference at the $5 \%$ level 


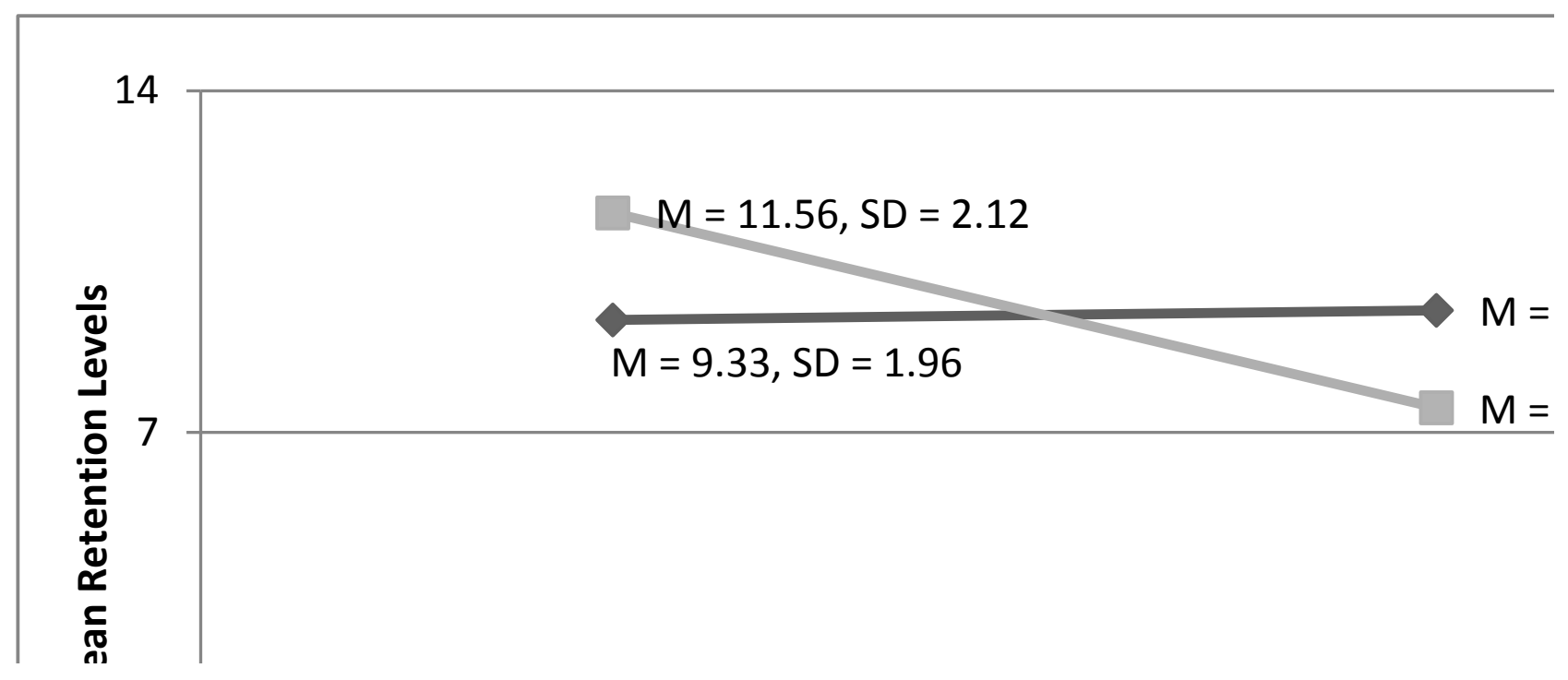

Figure 1. Comparison between E2 (Day 2) and E3 (Day 11) mean retention probe scores for symbol set 1 using Treatment 1 Self-Generation (T1 SG) and Treatment 2 Non-generation (T2 NG) 
Appendix A

Examples of the Connect-the-Dot Illustrations
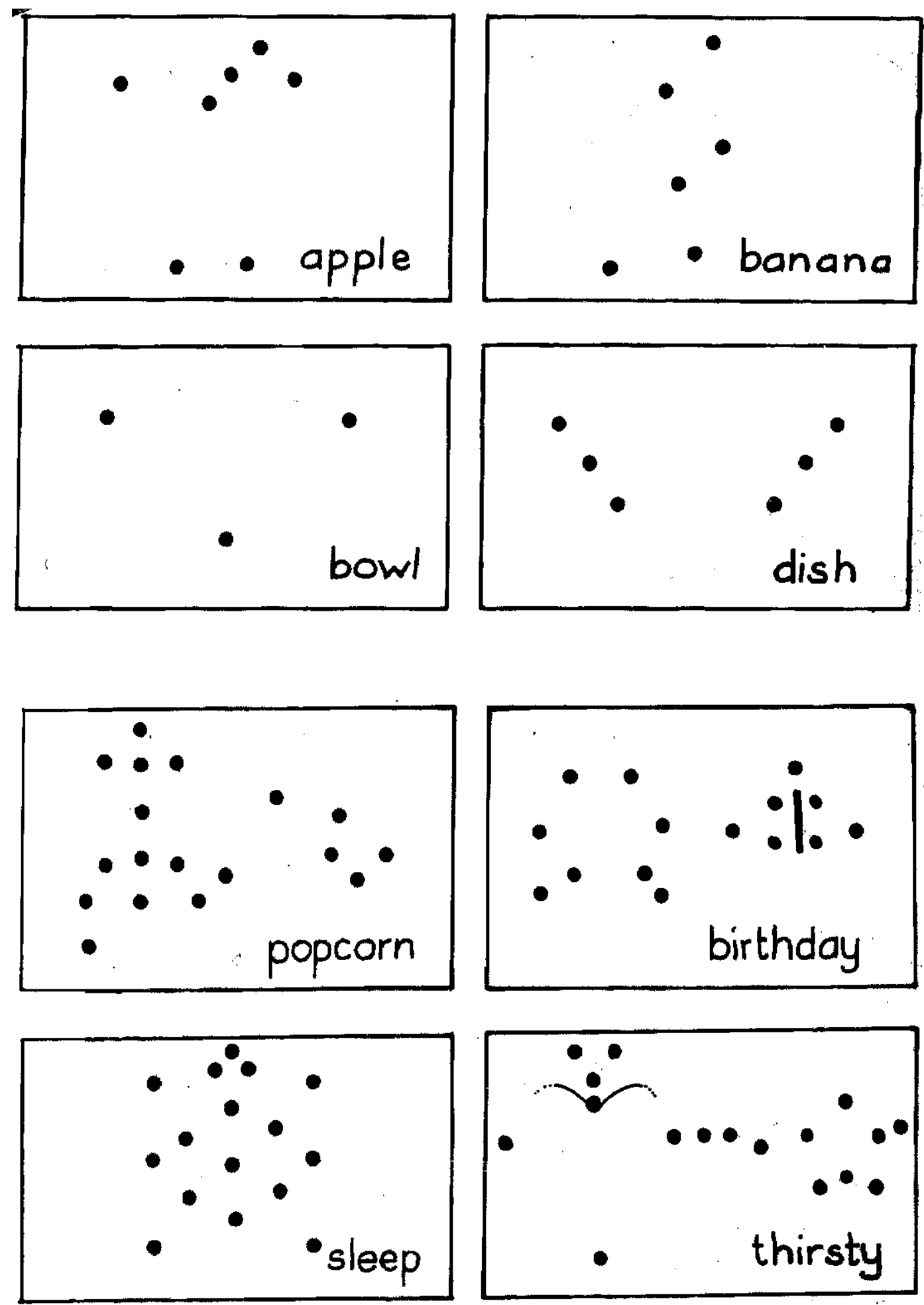